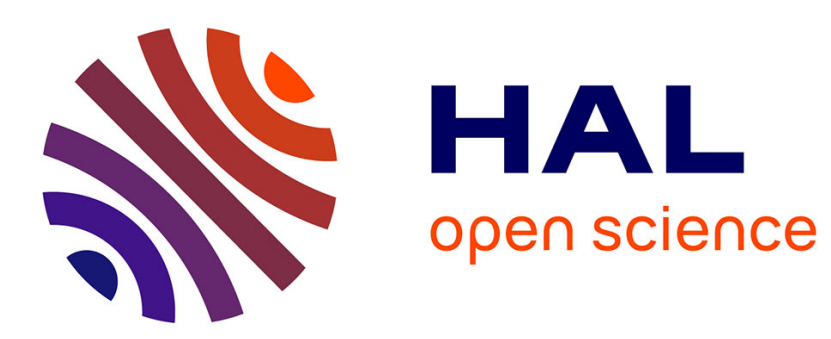

\title{
Hardening mechanisms in metals with foreign atoms
}

C. Schwink

\section{To cite this version:}

C. Schwink. Hardening mechanisms in metals with foreign atoms. Revue de Physique Appliquée, 1988, 23 (4), pp.395-404. 10.1051/rphysap:01988002304039500 . jpa-00245787

\section{HAL Id: jpa-00245787 https://hal.science/jpa-00245787}

Submitted on 1 Jan 1988

HAL is a multi-disciplinary open access archive for the deposit and dissemination of scientific research documents, whether they are published or not. The documents may come from teaching and research institutions in France or abroad, or from public or private research centers.
L'archive ouverte pluridisciplinaire HAL, est destinée au dépôt et à la diffusion de documents scientifiques de niveau recherche, publiés ou non, émanant des établissements d'enseignement et de recherche français ou étrangers, des laboratoires publics ou privés. 


\title{
Hardening mechanisms in metals with foreign atoms
}

\author{
C. Schwink
}

Institut für Metallphysik und Nukleare Festkörperphysik, Techn. Univ. Braunschweig, Mendelssohnstr. 3, 3300 Braunschweig, F.R.G.

(Reçu le 26 mai 1987, accepté le 6 juillet 1987)

\begin{abstract}
Résumé. - Nous faisons une revue des mécanismes pouvant empêcher et influencer les processus de glissement dans les métaux contenant des impuretés en solution ou sous forme de précipités. Après avoir rassemblé les interactions élémentaires, la contrainte de décrochage d'une dislocation est examinée; ses évaluations à $\mathrm{T}=0$ et les effets de la température sont résumés. Ensuite, le comportement d'ensemble de dislocations se déplaçant indépendamment ou bien en groupes est discuté pour des solutions solides dans le stade I ; l'intervention simultanée de mécanismes de durcissement et d'adoucissement est soulignée. Finalement, nous tenons compte des interactions dislocation-dislocation. En général, elles se superposent sur les interactions dislocation-impureté d'une façon qui n'est pas additive mais plutôt multiplicative.
\end{abstract}

\begin{abstract}
A survey of possible mechanisms impeding and influencing glide processes in metals with impurities (solutes and particles) is given. After putting together the elementary interactions, the threshold stress for a single dislocation is considered, its successful calculations for $T=0$ and the effects of thermal fluctuations are summarized. Then the behaviour of many dislocations moving either independently of each other or in correlated groups is discussed for solid solutions in stage $I$, the simultaneous occurrence of hardening and softening mechanisms in pointed out. Finally, the dislocation-dislocation interactions of strain hardening are taken into account. In general, they superimpose on the dislocation-impurity interactions not in a simply additive, but in a multiplicative way.
\end{abstract}

\section{Introduction}

This article deals with hardening mechanisms operating in metals containing impurity atoms. The impurities may be distributed in the matrix more or less at random as substitutional or interstitial solutes $/ 1,2,3 /$, or they may form a second phase of well-defined particles with diameters up to about hundred $\mathrm{nm}$ and a volume fraction not exceeding $10 \% / 4,5 /$ Two independent quantities are considered to fully characterize the plastic behaviour of any metal on a macroscopic scale:

i) The resolved flow stress $\tau$, starting at the critical resolved shear stress (crss) $\tau$ and increasing with strain a, as measured at a given temperature $T$ and strain rate $\dot{a}$, $\tau=\tau(a)_{T, \dot{a}}$, and

ii) the strain rate sensitivity of flow stress, $(\Delta \tau / \Delta \operatorname{lnå}) T^{\prime}$

as deduced from strain rate change or stress relaxation experiments.

The problem is firstly to find out possible elementary interactions between dislocations and impurities and then to interpret the macroscopic quantities by theories relying on these interactions. Many aspects and details of the problem are not yet satisfactorily solved. In the following just the principles of the solved and unsolved questions in this field will be sketched.

\section{Impurity induced lattice changes and elementary interactions}

At the outset, changes of structure and properties of an ideal lattice as induced by impurities are compiled which may in turn influence dislocation movement. Solute atoms and precipitate or dispersed particles will be considered in parallel.

II.1. Impurity induced lattice changes

1) Dilatation. By the impurity atoms the original volume of the matrix will be changed, in general. Around a particle one finds a corresponding local volume change $\Delta V$ which produces an elastic strain field. In the simplest case of solid solutions with fcc structures the average lattice dilation is adequately described 
by the misfit parameter $\delta$, $\delta=1 / \bar{a} \cdot d \bar{a} / d c$

$(\bar{a}=$ lattice constant, $\mathbf{c}=$ solute concentration). In hcp structures the axis ratio may additionally depend on $c$, in bcc structures interstitial solutes play a role more important than substitutional ones, because they produce tetragonal lattice distortions.

2) Modulus effects. Together with the atomic distances also the coupling potentials between the atoms change, characterized macroscopically by change of the elastic moduli. The shear modulus $\mu$, e.g. may differ for a precipitate particle from that of the matrix by $\Delta \mu$. For solid solutions the average quantity analogous to $\delta$ is the "elastic polarizability" $n$, $\eta=1 / \mu \cdot d \mu / d c / 6 /$.

3) Atomic ordering. As interatomic forces between atoms of different species are different, in general, the configurational energy can be minimized by segregation or ordering. Thus, in the case of particles coherent precipitates may possess an ordered atomic structure, in solid solutions short-range-ordered (SRO) or short-range-segregated (SRS) regions may form.

of course, coherent precipitates without ordering also represent regions differing from the surrounding matrix for a moving dislocation /5/.

4) Stacking fault energy (SFE). In connection with plastic properties this quantity deserves particular interest. On the one hand the SFE's of particles, $\gamma_{P}$, and of matrix, $\gamma_{M}$, may be different, on the other hand the SFE of a solid solution varies in general with solute content. The approach of a phase boundary of a system with increasing $c$ usually causes a decrease of $\gamma, \Delta \gamma / \Delta c<0$.

5) Interfacial energy. Interfaces between a matrix and particles, and energies connected with them are specific for the "particle case".

Any of these deviations from the properties of an ideal lattice produced by impurities will influence the motion of a dislocation and give rise to an interaction energy and to a force which adopts a specific maximal value $F_{m}$ in each case.

Tracing back the underlying processes, all of these interactions virtually are of electronic origin involving the conduction and valence electrons of host and foreign atoms. Therefore, they are correctly treated only by quantum theory. However, dislocation theory in metals is dominated by the theory of elasticity which yields practically correct results if the immediate neighbourhood (2-3b, $\mathrm{b}=$ Burgers vector) of dislocation core and foreign atoms is excluded in the calculations.

The reason for the success of this procedure is that the main part of the interaction energy is contained in the strain field far away from the strain centres and not in their atomic neighbourhood.

Effects of inevitably pure electronic origin, like SFE and interface energies, are taken into account by the use of experimental values for these quantities entering the formulae as parameters.

\section{II.2. Resulting elementary interactions $\mid 1,2,4,5 /$}

To 1) and 2). Interaction energies connected with dilatation and modulus effect originate from the strain fields around a dislocation line and a solute atom or particle which tend to approach a state of smallest elastic energy. The calculation of this "coherency-strain strengthening" is in the case of particles quite a difficult problem, in general $/ 4,5 /$. It is easier to solve for single solute atoms. However, for distances between dislocations and single solutes less than a few atomic distances an "electrostatic" (= electronic) contribution to the energy, which originates from differences in the valency of host and solute, has to be taken into account /1,2/. Estimates yield this "electrostatic energy" not to exceed about $1 / 5$ of the elastic one /2/, a complete theory is still missing.

To 3). A dislocation crossing a region of atomic order disturbs this order and creates on the glide plane an area of a specific boundary energy. By that an antiphase boundary of energy density $\gamma_{A P B}$ is developing in a coherent particle. Quite similarly, regions of $\mathrm{SRO}$ or SRS in solid solutions sheared off by dislocations give rise to boundary energies /7/. The occurrence of dislocation pairing reducing this energy has to be considered carefully when applying this interaction mechanism $/ 2,4,5 /$.

To 4). In fcc metals the SFE determines via the line energy of dislocations the extent of their splitting into partials. If a dislocation moves in a fcc matrix with fcc particles of a different SFE present, it will experience an attractive or repulsive force. In a solid solution the SFE may be diminished if the fault between the partials contains an (equilibrium) concentration of foreign atoms different from the average one. In this way, a persistent interaction energy between dislocations and solutes distributed non-randomly arises provided that the concentration difference between matrix and SFE is continuously maintained. Otherwise, at least a timedependent energy and force results $/ 1,2,8 /$.

To 5). A dislocation shearing off a coherent particle creates an extra surface between particle and matrix in the form of ledges of width $b$ with an interface energy $\gamma_{s} / 4,5 /$.

II.3. Examples and values

The preceding section was to show that the possible elementary interaction mechanisms between a dislocation and solutes or particles virtually are the same. To be more specific now the 
maximum interaction forces for characteristic examples are given.

In solid solution hardening the volume effect plays an important role. A single foreign atom producing a spherical dilatation changes the volume of the lattice by

$$
\Delta \mathrm{V}=3 \mathrm{~V}_{\mathrm{A}} \cdot \delta, \mathrm{V}_{\mathrm{A}}=\text { atomic volume. }
$$

It interacts most strongly with the strain field of an edge dislocation. If the position of the foreign atom is given by $(r, \psi)$ with respect to a straight edge dislocation the interaction energy is $/ 1 /$

$\Delta \mathrm{E}=3 / \pi \cdot \mu \mathrm{b} \cdot \mathrm{V}_{\mathrm{A}} \cdot \delta \cdot \sin \psi / \mathrm{r}$,

yielding numerical values of up to

$0,3 \mathrm{eV}$. For the maximum interaction

force one finds $F_{m}^{\Delta V} \approx \mu b^{2} \cdot \delta$, attaining

values of about $1 \cdot 10^{-10} \mathrm{~N} \quad\left(=1 \cdot 10^{-5}\right.$ dynes $)$ for $\mathrm{Cu}$-alloys with $\delta=0,04$.

In precipitation hardening the maximum interaction forces for the cases

i) of an atomic order destroyed across a glide plane of a particle of radius $\mathrm{R}$, and ii) of a freshly created interface area of width b are simply

$$
F_{m}^{A P B}=2 \gamma_{A P B} \cdot R \text {, and } F_{m}^{S}=2 \gamma_{S} \cdot b \text {, }
$$

respectively.

Characteristic values /4/ are

$\gamma_{\mathrm{APB}}=100 \mathrm{mJm}^{-2}, \gamma_{\mathrm{S}}=10 \ldots 100 \mathrm{mJm}^{-2}$

and $\mathrm{R}=100 \mathrm{~A}$, yielding

$\mathrm{F}_{\mathrm{m}}^{\mathrm{APB}}=2 \cdot 10^{-9} \mathrm{~N}$ and

$\mathrm{F}_{\mathrm{m}}^{\mathrm{S}}=5 \cdot 10^{-12} \ldots 5 \cdot 10^{-11} \mathrm{~N}$.

The interaction forces for precipitate particles following from the elementary mechanisms 1),2) and 4) yield dependences of $F_{m}$ on the particle radius $R$ which are in nom case stronger than linear $/ 4,5 /$. For the breaking strength of incoherent (dispersed) small particles also a simple proportionality, $F_{m} \propto R$, results /4/. The full calculation of the interaction forces in all cases mentioned is a difficult task and relevant problems still have to be solved.

The section is ended by the remark that a dislocation always experiences several of the different elementary interactions simultaneously. Their correct superposition is a problem in itself. To simplify the following discussion a single maximum interaction force is assumed to exist which governs the dislocation glide process.

\section{The threshold stress for motion of} a single dislocation

A single dislocation is assumed to move across a field of statistically distribut- ed obstacles. At a certain minimum stress, the threshold stress, it will begin to glide across the whole slip plane. The obstacles may be.singles, pairs or multiplets of foreign atoms, or precipitate or dispersed particles.

\section{III.1. Point obstacles}

To calculate the threshold stress we take the most simple case: The obstacles are considered as point-like and are overcome at $\mathrm{T}=0$, i.e. without any thermal activation.

The interaction force $\mathrm{F}$ between a dislocation and a single obstacle is favourably characterized by an angle $\theta$

(Fig. la), with $\mathrm{F}=2 \mathrm{~T} \sin \theta$, and $\mathrm{T}_{\mathrm{I}}$ representing the line tension,

$$
\mathrm{T}_{\mathrm{L}} \approx \mu \mathrm{b}^{2} / 2 \text {. }
$$

The maximum strength $F_{m}$ of obstacles of the same kind is connected with a breaking angle $\theta_{C}$ according to $F_{m}=\mu b^{2} \sin \theta_{C}$.

Without any external stress the dislocation is a straight line $(\theta=0)$ touching two point obstacles at most. With increasing stress it bows out, meets more and more obstacles (Fig. 1b) breaking through those for which $\theta>\theta_{C}$.

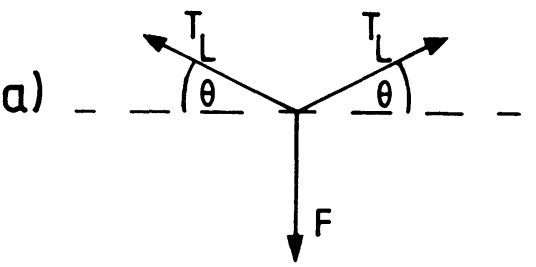

b)

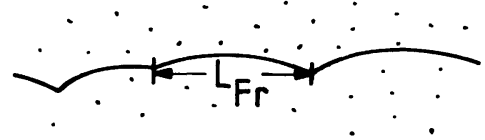

Fig. 1.a) Balance of forces at a point obstacle

b) Friedel length $\mathrm{L}_{\mathrm{Fr}}$

The problem to determine the average distance of anchoring points along the dislocation which is pushed by a stress $\tau$ against the obstacles has been solved by Friedel /9/ in an approximation which proved very powerful:

$$
I_{F r}=\left(2 T_{L} b / \tau c\right)^{1 / 3}=\left(\mu b_{O}^{2} / \tau\right)^{1 / 3} .
$$

Here $\mathrm{c}$ is the obstacle concentration per atom and $\mathrm{L}_{\mathrm{O}}=\mathrm{b} / \mathrm{c}^{1 / 2}$ the average obstacle distance.

The critical minimum stress $\tau$ is obtained applying Fleischers /10/ average balance of forces,

$$
\mathrm{F}_{\mathrm{m}}=\tau_{\mathrm{c}} \mathrm{bL}_{\mathrm{Fr}} \text {. }
$$


Combining the last equations yields the well-known relation for the threshold stress:

$$
\begin{aligned}
\tau_{C} & =\mu b\left(\sin \theta_{C}\right)^{3 / 2} / L_{o} \\
& =\mu\left(\sin \theta_{C}\right)^{3 / 2} c^{1 / 2},
\end{aligned}
$$

tor $0 \leqq \theta_{C} \leqslant 40^{\circ}$.

Equ. (1), showing immediately how $\tau_{c}$ increases with obstacle strength and concentration, is valid for sufficiently low breaking angles $\theta$. Computer simulations /11/ revealed the rather wide range of validity as given above and yielded additionally an approximate relation for larger $\theta_{C}$ (cf. also /5/):

$$
\begin{aligned}
\tau_{C} & =0,8 \mu \mathrm{b} \sin \theta_{C} / L_{o} \\
& =0,8 \mu \sin \theta_{C} \cdot c^{1 / 2},
\end{aligned}
$$

for $40^{\circ} \leqslant \theta_{C} \leq \pi / 2$.

\section{III.2. Particles /4,5/}

Equ. (2) is of importance for particle strengthening where for impenetrable particles even $\sin \theta=1$ is realized.

To improve the apparently unjustified assumption of particles to represent point obstacles, the case of spherical particles of radius $R$ occupying a volume fraction $f$ is considered. For a flexible dislocation line the particle distance $\mathrm{L}$, now dependent also on $\mathrm{R}$, to a quite good approximation /4/ is:

$$
\mathrm{L}_{\mathrm{O}}=(2 / \mathrm{f})^{1 / 2} \cdot \mathrm{R} \text {. }
$$

Further, for a given obstacle strength $\mathrm{F}_{\mathrm{m}}$ the maximum stress $\tau$ which a particle $m$ of radius $R$ can exert ${ }^{m}$ on the dislocation is:

$$
\tau_{\mathrm{m}}=\mathrm{F}_{\mathrm{m}} / 2 \mathrm{Rb} \text {. }
$$

Equs. (1) and (2) for the critical stress now become (with $f$ considered as constant) :

$$
\tau_{C}=2 \tau_{m} f^{1 / 2}\left(R \tau_{m} / \mu b\right)^{1 / 2},
$$

for $0 \leqq R \leqq 0,32 \mu \mathrm{b} / \tau_{\mathrm{m}}$;

$$
\tau_{c}=1,13 \tau_{m} f^{1 / 2} \text {, }
$$

for $0,32 \mu b / \tau_{m} \leqslant R \leqq \mu b / 2 \tau_{m}$.

Particles with $R \geqq \mu b / 2 \tau$ cannot be penetrated by the dislocation. Rather, after passing the obstacle the dislocation leaves an Orowan-loop around the particle (Fig. 2). The breaking angle $\theta$ is permanently $\pi / 2$ now, independent of $R$, and the critical stress becomes equal to the Orowan-stress:

$$
\tau_{C}=0,8 \mu \mathrm{b} / \mathrm{L}_{\mathrm{O}}=0,56 \mu \mathrm{bf}^{1 / 2} / \mathrm{R},
$$

for $R \geqq \mu b / 2 \tau_{m}$.

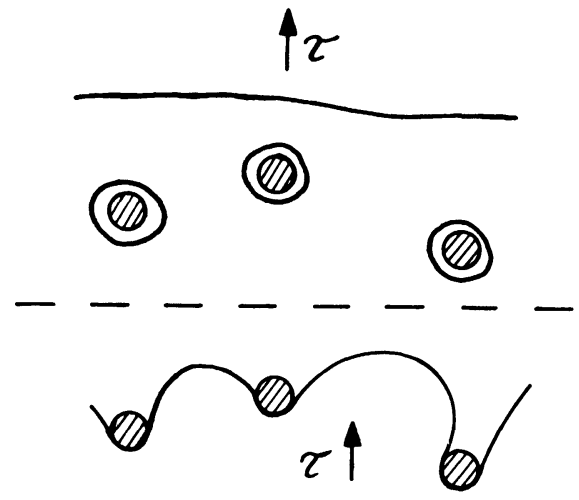

Fig. 2. A dislocation passing impenetrable particles leaves behind orowan-loops

In most age-hardening systems the radius $R$ of the precipitates increases by competitive growth at constant volume fraction $f$. Therefore, equs. (1') to (3') describe correctly the qualitative behaviour of the critical resolved shear stress (crss) as dependent on R:

For small particles, as long as equ. (1')

applies, $\tau_{\text {}}$ increases proportional to $\mathrm{R}^{1 / 2}$. Particles $C$ within the range of size of equ. (2') are cut with a stress depending only on obstacle strength and volume fraction, but not on the particle size.

Very large particles, finally, are not sheared off by the dislocation, but enclosed by loops. The crss is now independent of $\tau_{m}$ and, therefore, of the properties of the particle, it decreases with increasing radius. Such alloys are called "over-aged".

Experiments show that the described principles which neglect, among other details, any thermal fluctuations explain the main features of age-hardening systems. The main reason that in most cases an appreciable influence on $\tau$ arising from thermal activation is missing is the size of the particles. If their cutting is hindered by effects of stacking fault or interface energy, it could appreciably be supported by thermal energy only if the energy barriers were rather small, certainly less than $2 \mathrm{eV}$ (cf. IV.1.), and of atomic dimensions. On the other side, the hardening mechanisms based on lattice dilatation and modulus effects depend on temperature only via thermal expansion, i.e. rather weakly, too.

III.3. Solid solutions /1,2,3/

In solid solutions the glide obstacles formed by the foreign atoms are always weak ones, $\mathrm{F}_{\mathrm{m}} \ll 2 \mathrm{~T}_{\mathrm{L}}$. Besides, the strength they are characterized by their 
average distance $l_{b}$ on a glide plane and by the width $w$ representing the range of interaction between obstacle and dislocation $(w=2-3 b / 12,13 /)$. The value of the dimensionless paramater $\beta / 14,15 /$,

$$
\beta=\left(w / I_{b}\right) \cdot\left(2 T_{L} / F_{m}\right)^{1 / 2},
$$

indicates whether the glide barriers in solid soli:tions represent point obstacles or not. The first factor in $\beta$ is a measure of the localization of the defects, the second one of their strength, in real cases

$\left(2 \mathrm{~T}_{\mathrm{L}} / \mathrm{F}_{\mathrm{m}}\right)^{1 / 2}$ taking values between 3 to 10 , approximately.

The case $\beta \leqq 1$ means that the solid solution is dilute, $l_{b}>w$. Then for a stationary gliding dislocation the defects act as point obstacles opposing its motion. The critical stress at $\mathrm{T}=0, \tau$, has to be calculated using "Friedel statistics", equ. (1), yielding (cf. /2/):

$$
\tau_{\mathrm{OO}}=\mathrm{F}_{\mathrm{m}}^{3 / 2} \mathrm{c}^{1 / 2}\left(2 \mathrm{~T}_{\mathrm{L}} \mathrm{b}^{4}\right)^{-1 / 2} .
$$

With increasing obstacle concentration $\mathrm{c}$ this Fleischer-Friedel formula /2/ changes into:

$$
\tau_{\text {oo }}=\alpha c^{1 / 2}\left(1+\beta^{\prime} c^{1 / 2}\right) / 12 / \text {. }
$$

The case $\beta \geqslant 0,5$ means that the solid solution is more concentrated, though still $l_{b}>w$, and that additionally the obstacles are relatively weak ( $F_{m}$ low) as compared to the situation of equ. $(1 ")$. In the case $\beta \geqslant 0,5$ which was thoroughly analyzed by Labusch $/ 14,16 /$ a stationary gliding dislocation not only interacts with barriers at its front flanks, but also with those at its back flanks. Thus, it experiences stresses caused by the defects in the same direction as well as in the opposite one of an applied stress. Now, the calculation of $\tau_{00}$ follows another statitistics, called "Mott-Labusch statistics" yielding

(4) $\tau_{o O}=\mathrm{F}_{\mathrm{m}}^{4 / 3} \mathrm{c}^{2 / 3}(\mathrm{w} / \mathrm{b})^{1 / 3}\left(4 \mathrm{~T}_{\mathrm{L}} \mathrm{b}^{6}\right)^{-1 / 3}$.

The exponents for the interesting quantities $F_{m}$ and $c$ have changed slightly as compared to equ. (1"). The superposition of the effects of different obstacles on the same dislocation are taken into account by an effective obstacle strength, $\mathrm{F}_{\mathrm{m}}^{\prime}$, instead of $\mathrm{F}_{\mathrm{m}} / 2 /$.

The regimes of Friedel- and Mott-Labusch-statistics overlap for a certain interval of $\beta$-values $/ 12 /$, as indicated above.

Stationary dislocation glide means that the moving dislocation overcomes the defect potentials by passing quasistatically from one equilibrium configuration to the next one. That presumes viscous forces to act on the dislocation in such a way that its motion is "overdamped". At higher temperatures interactions with phonons guarantee always such a type of motion. However, at sufficiently low temperatures, and in particular at $\mathrm{T}=0$, in dilute alloys the dislocation motion may become "underdamped" which induces "inertial effects" /17,18/:

An underdamped dislocation approaching an obstacle with increasing velocity may overshoot the next static equilibrium position exerting a force on the obstacle which is greater than the maximum force in the equilibrium case. By that it may overcome the next and any following obstacle on impact at a stress less than $\tau_{\text {oo }}$ of equs. (1") or (4). This softening mechanism has been completely incorporated into a theory of solution hardening at $\mathrm{T}=0 / 12 /$.

At $\mathrm{T}=0$ defect obstacles may be crossed by a dislocation with a stress less than $\tau$ oo even if it is moving quasi-statically. That happens if the dislocation motion is essentially governed by the laws of quantum mechanics. Two possible quantum effects are discussed in literature, tunnelling of a dislocation line and its vibrations at $\mathrm{T}=0$. In both cases quantum mechanics calculates the probability for the dislocation to cross a defect potential by passing a barrier potential, either by the direct tunnelling of it or by the quantum vibrations "lowering" the top of the potential /19,20/.

Both quantum mechanical modes of motion represent softening mechanisms, too, resulting in a reduction of $\tau_{00}$.

The relative deviation of the threshold stress, $\Delta \tau_{0 \circ} / \tau_{0 \circ}$ ' as induced by these effects may be taken as a quantitative measure. From experiments one can deduce as rough estimates /27/

$\Delta \tau_{00} / \tau_{\text {oo }}=0,10$ for the zero-point $v i-$ bration effect in concentrated $\mathrm{Cu} \mathrm{Mn}$, and

$\Delta \tau_{\text {OO }} / \tau_{\text {OO }}=0,3-0,7$ for the inertial effects of different dilute Cu-alloys, the ratio decreasing with increasing solute content. +)

III.4. Effects of thermal fluctuations /21/

While the main features of particle hardening which appear at higher temperatures can be virtually understood by considering the glide processes at $T=0$, the same is not true for solid solutions. The obstacles are now of atomic size (singles, pairs or multiplets of foreign atoms) with barrier energies of about $1 \mathrm{eV}$, typically. Therefore, thermal fluctuations will appreciably support the dislocations to overcome the obstacle barriers.

The effect of thermal activation can be safely described $/ 20 /$ by an Arrhenius-equation for the transition rate $v$ of the dislocation:

$$
\nu=\nu_{0} \exp (-\Delta G(\tau) / k T) .
$$

+ ) See note 1. at the end 
$\nu_{0}$ is an attack frequency and $\Delta G$ the activation enthalpy of the barrier $/ 21 / \mathrm{de}-$ creasing if a stress $\tau$ is applied.

The exact path of a dislocation driven by an applied stress may be drastically changed by thermal fluctuations. Their essential effects up to about $400 \mathrm{~K}$, however, can be easily visualized /12/: i) Above about $30 \mathrm{~K}$ the viscous damping strongly increases inducing a permanently quasi-static dislocation motion.

ii) The height of all barrier potentials appears as if lowered in such a way that the decreasing waiting times of the dislocations in front of the obstacles remain the same for all activation events, on the average. The resultant flow stress reducing effect will be further analysed in connection with experiments.

Above $400 \mathrm{~K}$, or even below, another different elementary mechanism influencing dislocation movement comes into play. The foreign atoms near a dislocation no longer remain at fixed positions, rather they tend to lower their energy by diffusing across atomic distances in the stress field of the dislocation. Clinging to the dislocation they impede its motion. The observation of this effect of stress induced diffusion depends on the timescale of the method of measurement applied, the time of reference always being the waiting time of the moving dislocations in front of the obstacles. In measurements of the crss, $\tau(T)$, the time-scale of experiment is determined by the inverse of the strain rate. In measurements of stress relaxation, $t(t)$, the upper limit of measuring time, depending on the minimum resolvable $\Delta \tau$ at constant conditions, may attain hours. Correspondingly, in the first case the diffusion effect as indicated by serrations in the $\tau(a)$ curve $/ 22 /$ (Portevin-LeChatelier effect) clearly appears between $400 \mathrm{~K} \leqslant \mathrm{~T} \leqslant 700 \mathrm{~K}$, while in the second case displacements of foreign atoms could be detected already at rather low temperatures down to about $\mathrm{T}_{\mathrm{m}} / 5\left(\mathrm{~T}_{\mathrm{m}}=\right.$ melting point $)$ $123,24 /$.

Increasing the temperature the foreign atoms become mobile enough to lock the dislocations during their waiting at stronger obstacles thus impeding their motion rather effectively.

At still higher temperatures, $T \approx 700 \mathrm{~K}$, one finally enters the regime of free diffusion of foreign atoms. They no longer form obstacles for the dislocation which now can move at very small stresses.

\section{$\frac{\text { IV. Solid solution hardening (crss) }}{\mid 1,2,3,13 /}$}

measurable crss, even if extrapolated to $T=0$. In the preceding chapter only the case of a single dislocation gliding across a distribution of obstacles has been considered. Mostly, however, large numbers of dislocations are moving simultaneously. That provides the possibility that a variable number of inhomogeneously arranged dislocations may circumvent the obstacles at a lower stress than a single one.

The following considerations will be confined to fCC systems where the largest amount of information is available.

The question whether and how the real crss of solid solutions may deviate from the threshold stress will be discussed for three cases:

i) All mobile dislocations are moving nearly independently of each other.

ii) Groups of dislocations moving strongly correlated are formed from the very beginning of deformation.

iii) The flow stress contribution of the density of immobile dislocations which rapidly increases during deformation becomes comparable to that of the defect obstacles (whereas the contribution of the grown-in dislocation density may be negligible in any of the three cases).

Point iii) will be discussed separately in sect. $\mathrm{V}$.

\section{IV.1. Independently moving dislocations}

Case 1 evidently is the most simple one, because here threshold stress and crss should immediately be comparable. To realize it the formation of correlated dislocation groups has to be avoided. That is achieved by choosing a system with a negligibly small tendency for SRO (see IV.2.) which is rather well accomplished for CuMn, e.g., at least up to 5 atomn.

The temperature dependence of the crss (Fig. 3) for a set of Mn-concentrations shows: Not only at low concentrations (c< 2atoMn) where the curves are qualitatively similar to those of other systems $125 /$, but also at higher ones the low temperature anomalies (beginning around a negative curvature of $\tau(T))$ prevent a safe extrapolation of $\tau_{0}(T){ }^{\circ}$ to $T=0$. That would presuppose a theory comprehending thermal activation as well as inertial effects to exist. As it is lacking an immediate proof of equs. (1") and (4) by experiment is not yet feasible.

A comparison with theory in the interval $80 \mathrm{~K} \leqslant \mathrm{~T} \leqslant 400 \mathrm{~K}$, the regime of prevailing thermally activated processes is at time only possible if a system follows Friedel statistics (Mott-Labusch statistics including thermal activation is just being developed /26/). Then the Arrhenius-equation as forumlated for the strain rate,
The threshold stress of solid solutions as considered in sect.III.3. must not immediately be identified with the $\dot{a}=\dot{a}_{0} \exp \left(-\Delta G\left(\tau_{0}\right) / k T\right)$ 
yields, according to the lines sketched in sect.III., the crss $\tau(T)$ as function of temperature $/ 1,21 /$. The average activation enthalpy, $\Delta G$, has to be deduced from a physically reasonable defect potential, e.g. Cottrell-Bilby's, the constancy of a has to be examined by experiment (See IV.2.). The theory contains only 2 parameters, obstacle strength $F_{m}$, and obstacle concentration $c_{b}$ (whichmay

be different from the concentration of foreign atoms!), if the obstacle width $\mathrm{w}$ is taken from reliable estimates $/ 12,13 /$ as $\mathrm{w}=2,5 \mathrm{~b}$ (see III.3.). By combining the two independent sets of data accessible. to experiments, $\tau_{0}(T)$ and activation volume $\mathrm{V}(\mathrm{T})$, one obtains these parameters with good accuracy if the experiments are done on the same crystals oriented for single glide /27/.+)

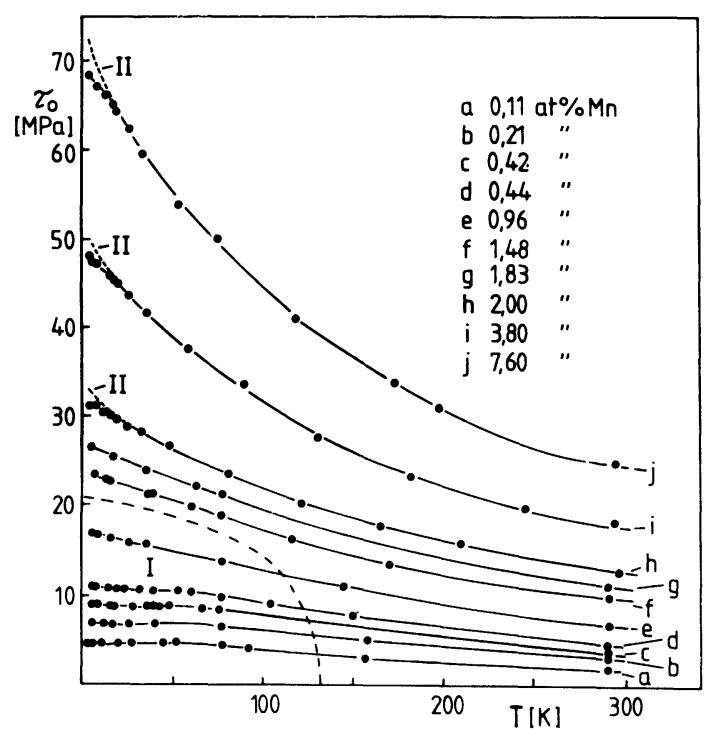

Fig. 3. The crss, $\tau(T)$, for single crystals of ${ }^{\circ}$ CuMn-alloys oriented for single silip as a function of temperature. I is to indicate the regime of inertial effects, II probably that of zero-point vibrations /27/

For the following reasons CuMn proved to be a system well suitable to apply the Fleischer-Friedel theory:

i) The misfit parameter, $\delta=0,11$, is very high as compared to other $\mathrm{Cu}$-based systems. That increases the chance of formation of point-like obstacles. ii) The SFE does not change with Mn-concentration, at least up to 12 atomn $/ 28 /$. Including the lack of SRO up to 5 at:Mn one finds that the possible elementary mechanisms of dislocation-obstacle barrier interactions are reduced to a minimum.

In fact, a corresponding analysis of the CuMn-data proved possible yielding

\footnotetext{
+) See note 2. at the end
}

activation enthalpies of about $1,2 \mathrm{eV}$ and obstacle densities of about $5 \%$ of the whole Mn-content. The results suggest that mainly Mn-pairs are the relevant glide barriers. Furthermore, an athermal stress component independent of temperature results which is probably due to multiplets of Mn-atoms. The Labuschparameter $\beta$ turns out smaller than 0,2 for all concentrations justifying the application of Friedel-statistics /27/.

\section{IV.2. Correlated dislocation groups}

Large groups of parallel dislocations of the same sign are observed already in stage $I$ of deformation at higher solute concentrations (c $\geqslant 2-5 a t \%)$, in general. In systems like CuAl, CuGe, Cuzn the SFE rapidly decreases with impurity concentration $/ 2 /$. For this reason it was believed, at first, that the increased width of extended dislocations impeding cross slip causes the occurrence of planar glide. The latter was detected, however, also in systems with high SFE, as disordered $\mathrm{Ni}_{3} \mathrm{Fe} / 29 /$,

and with SFE not changed in comparison with the pure metal, as Cu-12atomn /28/. In both systems SRO occurs, as does in the above-mentioned other alloys, too. These results strongly suggest that in solid solutions the SRO regions are responsible for effectively impeding cross slipping which in turn favours the development of planar groups.

These are kept together because the front dislocations experience a larger resistance than the following ones owing to the shearing of the SRO regions.

Growth and movement of processions of up to serveral hundred dislocations can be immediately observed and investigated by microcinematography $/ 30 /$. It revealed that increasing the strain rate increases the number of simultaneously mobile dislocation groups which experimentally becomes apparent and measurable as an increased volume of all simultaneously active slip zones taken together. The change of this "active slip volume" /31/ formally means that the preexponential $\dot{a}_{0}$ in the Arrhenius-equation becomes dependent on strain rate. As a consequence, the increase in flow stress with increasing strain rate, $(\Delta \tau / \Delta \operatorname{lna})_{T}{ }^{\prime}$ is

lower than it were for constant active slip volume, and it no longer represents the (inverse) activation volume. The thermal activation analysis corrected for this effect yields changed activation parameters $122 /$, e.g. lower activation enthalpies. As a whole, the variability of the active slip volume with strain rate is an elementary dynamical mechanism reducing the increase of flow stress up to a factor of 2 .

The large stresses around these dislocation groups are for the most part compensated by the formation of multipoles of edge dislocations as familiar from TEM pictures of concentrated fcc alloys. The evaluation of the arrangement of sta- 
tic pile-ups in $\mathrm{Ni}-30 \mathrm{wt} \% \mathrm{Cr}$ alloys /32/ revealed for the front dislocations (even in the thin TEM foils!) local stress concentrations of $2-3$ times the crss. Similar values were found in $\mathrm{Cu}$ alloys for the driving stress for two dislocation groups to form a multipole /33/.

These results involve some difficult problems. Since the dislocation groups and multipoles develop already in the first stage of deformation the question arises whether the crss as determined from $\tau(a)-$ curves has to be identified with the average peak stress necessary to activate the very first dislocation of each group, or with the average stress necessary to move the groups. In the first case the considerations of the threshold stress for a single dislocation (including source stresses) apply, in the second case the complicated elastic stress field of groups and multipoles has to be taken into account.

The fact that the concentration dependences of the crss quite continuously pass the region where planar slip sets in /1,2/ favours the first view, at first sight. However, there are strong arguments against it:

i) Within the completely smooth easy glide range of deformation practically the total strain comes from the motion of the groups determining, therefore, the stress level of stage $I$ and with it the crss. ii) As frequently observed - on EM replicas as well as by microcinematography /30/ slip in stage I proceeds by a transfer of glide processes from previously active slip zones to new ones, e.g. by double cross slip /34/ of dislocation segments. Based on pulse load experiments on $\mathrm{CuNi} / 35 /$ transfer mechanisms of glide from activated to fresh slip planes were recently taken to be responsible for the value of crss, too.

In this case dislocations are inevitably moving strongly influenced by the stress fields of neighbouring dislocations.

Theories of the crss based on the dislocation motion as influenced by elastic interaction with dislocation groups, or taking account of transfer processes of glide do not exist.

\section{Strain hardening}

Most investigations of age hardening and solid solution hardening deal with the increase of the yield stress and with the beginning of deformation. Much less studies exist /36/ on the general strain hardening behaviour leaving a lot of problems unsolved as yet.

With proceeding deformation the given number of glide obstacles formed by the foreign atoms is increased by those which are formed by the continuously generated dislocations. Both types of obstacles will contribute to the total flow stress.
Different laws of superposition have been proposed /37/ the limiting cases being the linear and the quadratic superposition, resp.:

$$
\begin{aligned}
& \tau=\tau_{1}+\tau_{2}, \\
& \tau^{2}=\tau_{1}{ }^{2}+\tau_{2}{ }^{2} .
\end{aligned}
$$

If a small number of strong obstacles is added to a large number of weak ones the corresponding stresses add up independently and the linear law applies. If the strength of the large number of present obstacles is comparable to that of the added ones, the densities of obstacles add and the quadratic law is approached $/ 36,37 /$.

Thus, the linear law should hold if the effects of solute defects and dislocations are superimposed $/ 36 /$. An experimental test for that is $/ 36 /$ that

$$
(\Delta \tau / \Delta \operatorname{lnå}) \text { plotted vs. } \tau \text {, }
$$

the Haasen-plot $/ 38 /$, yields a straight line. If the effect of the foreign atoms would be completely described by the linear law, the $\tau(a)$-curves of the alloys would simply be shifted by $\tau$ parallel to the $\tau(a)$-curve of the pure matrix.

Various experiments on polycrystals point out a different behaviour /36/. Fig. 4 represents recent stress-strain curves for pure $\mathrm{Cu}$ and dilute CuMn crystals oriented along $\langle 100\rangle$. There is no apparent difference in the homogeneity of deformation between pure $\mathrm{Cu}$ and the alloys.

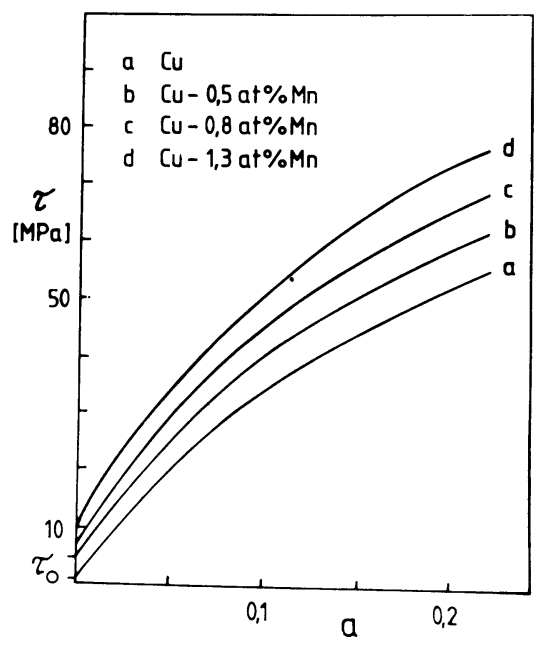

Fig. 4. Stress-strain curves for single crystals oriented along $\langle 100\rangle$ of pure $\mathrm{Cu}$ and dilute CuMn-alloys. By alloying the crss, $\tau$, as well as the strain hardening ${ }^{\circ}$ rate increases

Fig. 4 gives clear evidence that the strain hardening rate, d $\tau / d a$, increasing 
with solute content exceeds the values for pure $\mathrm{Cu}$ up to the onset of strain softening processes. That means that the solutes influence strain hardening not simply additively, but in a multiplicative way /36/ up to stresses several times the crss.

Different mechanisms which could be responsible for this behaviour were proposed /39/:

i) Precipitation of foreign atoms during deformation:

ii) variation of interaction strength between reacting dislocations;

iii) increased rate of dislocation multiplication during deformation;

iv) reduction of recovery and/or annihilation rates of dislocations.

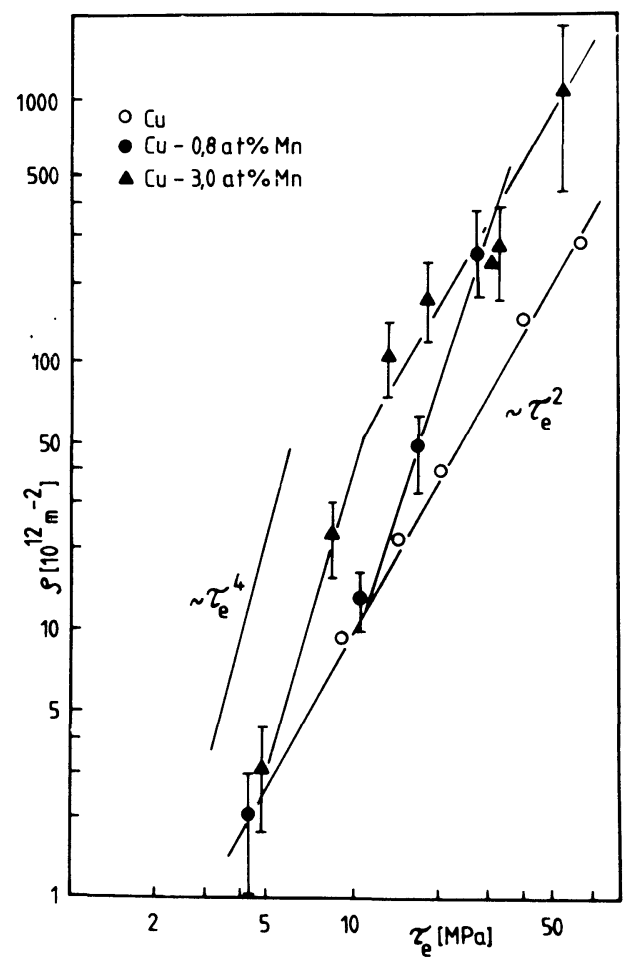

Fig. 5. Dislocation densities, $\rho$, as determined by TEM for single crystals oriented along $\langle 100\rangle$ of pure $\mathrm{Cu}$ $/ 41 /$ and of CuMn-alloys. Within an appreciable interval of 'effective' stresses, $\tau=\tau-\tau$ ' the increase of $\rho$ is much stronger than quadratic. Note the double-logarithmic plot.

As a first step towards a solution the investigation of the dislocation density revealed asurprising result $/ 40 /$. The total dislocation density $\rho$, plotted as function of the 'effective' stress seems to follow the familiar quadratic law at the beginning and towards the end of deformation. Within a certain appreciable range in between, however, $\rho$ increases much stronger approximately according to

$$
\rho \propto \tau e^{4}(\text { Fig. 5) },
$$

$\rho$ exceeding the pure $\mathrm{Cu}$ values at the same effective stress $\tau_{e}$ by a factor up to 7 .

In our view, this "storage phase" indicates a change of the mechanism of developing the dislocation structure governed by the foreign atoms.

\section{Conclusion}

In conclusion, we try to compile the interactions experienced by glide dislocations in plastically deforming alloys in a systematic way.

At the outset, there are the different elementary interactions between dislocations and the lattice changes induced by impurities. They all tend to retain gliding dislocations and are very high for true particle hardening. The main features of this case which is also of main practical importance are explained by these interactions refering to single dislocation motion at $\mathrm{T}=0$. In solid solutions, however, there are different ways for the dislocations to reduce the stress necessary for overcoming the obstacles.

In the most simple case of a single dislocation different softening effects exist already at $\mathrm{T}=0$ : The inertial effects in dilute alloys and quantum effects of motion, such as tunnelling and zero-point vibrations. At finite temperatures thermal activation is added as a further softening mechanism.

However, as soon as the mobility of the impurities increases, new hardening mechanisms come into play, like stress-induced diffusion processes and the dragging of impurities along with moving dislocations (Cottrell-clouds). Dynamic friction effects were omitted. Completely freely diffusing impurities impede the dislocation motion no longer, of course.

Interesting effects may appear in real cases where many dislocations are moving. On the one hand the inhomogeneity or nonuniformity of slip processes can effect a dependence of the total number of mobile dislocations on temperature and strain rate. On the other hand planar groups of dislocations and multipoles appear at higher impurity concentrations (c $\geqslant 2$ - 5 at\%) connected with intense elastic strain fields impeding as well as supporting the overcoming of obstacle distributions.

Multiplication and interactions of dislocations with each other as occurring during strain hardening depend on the impurities, in general. By that the superposition of impurity hardening and strain hardening is not simply a purely additive, but a multiplicative process.

In the sequence of the possible mechanisms described the number of unsolved problems increases. 
Acknowledgements. Useful comments by Prof. Neuhäuser are gratefully acknowledged. The experiments represented in Figs. 3-5 were supported by the DFG, project A8, SFB 319 .

\section{References}

/1/ Haasen, P., in "Dislocations in Solids" edited by F.R.N. Nabarro, Vol. 4, p. 156, North Holland, Amste $=$ dam, 1979.

/2/ Haasen, P., in "Physical Metallurgy" edited by R.W. Cahn and P. Haasen, p. 1341, North Holland, Amsterdam, 1983.

/3/ Suzuki, H., in "Strength of Metals and Alloys" edited by P.Haasen, V. Gerold and G. Kostorz, Vol. 3, p. 1595, Pergamon, Oxford 1980 .

/4/ Gerold, V., in "Dislocations in Solids" edited by F.R.N. Nabarro, Vol. 4, p. 220, North Holland, Amsterdam, 1979.

/5/ Brown, L.M., in "Strength of Metals and Alloys" edited by P. Haasen, V. Gerold and G. Kostorz, Vol.3, p. 1551, Pergamon, Oxford, 1980.

/6/ Kröner, E., Phys. Kondens. Materie 2 (1964) 262 .

/7/ Büchner, A.R. and Pitsch, W. Z. Metallkde. 76 (1985) 651 .

/8/ Suzuki, H., in "Dislocations and Mechanical Properties of Crystals" edited by J.C. Fisher, p. 361, Wiley, New York, 1957.

/9/ Friedel, J., Les Dislocations, Gauthier-Villars, Paris, 1956.

/10/ Fleischer, R.L., Acta Metall. 9 (1961) 996.

/11/ Foreman, A.J.E. and Makin, M.J., Phil.Mag. 14 (1966) 911.

/12/ Schwarz, R.B. and Labusch, R., J. Appl. Phys. 49 (1978) 5174 .

/13/ Kocks, U.F., Metall. Transactions A 16 (1985) 2109

/14/ Labusch, R., Phys. Stat. Sol. 41 (1970) 659 .

/15/ Nabarro, F.R.N., J. Less-Common Met. 28 (1972) 257.

/16/ Labusch, R., Acta Metall. 20 (1972) 917 .

/17/ Granato, A.V., Phys. Rev. B4 (1971) 2196.

/18/ Suenaga, M. and Galligan, J.M., Scripta Metall. 5 (1971) 829.

/19/ Natsik, V.D., Sov̄. J. Low Temp. Phys. 5 (1979) 191.

/20/ Labusch, R., Crystal Res. Technol. 19 (1984) 315 .

121/ Schoeck, G., in "Dislocations in Solids" edited by F.R.N. Nabarro, Vol. 3, p. 63, North Holland, Amsterdam, 1980.

/22/ Traub, H., Neuhäuser, H. and Schwink, Ch., Acta Metall. 25, (1977) 437, 1289.

/23/ Gleiter, H., Acta Metall. 16 (1968) 455,857 .
/24/ Neuhäuser, H. and Flor, H., Scripta Metall. 12 (1978) 443.

/25/ Basinski, Z.S., Foxall, R.A. and Pascual, R., Scripta Metall. 6 (1972) 807

/26/ Labusch, R., Verhandl. DPG (VI) 21 (1986) 1323.

/27/ Wille, Th. and Schwink, Ch., Acta Metall. 34 (1986) 1059; Wille, Th., Gieseke W. and Schwink, Ch., to appear in Acta Metall.

128/ Steffens, Th., Korner, A., Karnthaler, H.-P. and Schwink, Ch., Phil. Mag. (1987), in press.

/29/ Karnthaler, H.-P. and Schügerl, B., in "Strength of Metals and Alloys" edited by P. Haasen, V. Gerold and G. Kostorz, Vol. 1, p. 205, Pergamon, Oxford 1979.

$130 /$ Neuhäuser, H., in "Dislocations in Solids" edited by F.R.N. Nabarro, Vol. 6, p. 321 , North Holland, Amsterdam 1983.

/31/ Schwink, Ch., Phys. Stat. Sol. 18 (1966) 557.

132/ Clément, N., Caillard, D. and Martin, J.L., Acta Metali. 32 (1984) 961 .

/33/ Neuhäuser, H., Arkan, O.B. and Potthoff, H.-H., Mat. Sci. Eng. 81 (1986) 201.

134/ Jacksor, P.J., Progr. Mat. Sci. 29 (1985) 139.

135/ Neuhäuser, H. and Arkan, O.B., Phys.stat.Sol., in press.

/36/ Kocks, U.F., in "Strength of Metals and Alloys" edited by P. Haasen, V. Gerold and G. Kostorz, Vol. 3, p. 1661, Pergamon, Oxford 1980.

/37/ Kocks, U.F., Argon, A.S. and Ashby, M.F., Progr.Mat.Sci. 19 (1975) 224 .

/38/ Haasen, P., Phil. Mag. 3 (1958) 384 .

/39/ Schmidt, C.G. and Miller, A.K., Acta Metall. 30 (1982) 615.

/40/ Steffens, Th., Reip, C.-P. and Schwink, Ch., Scripta Metall. 21 (1987) March issue.

/41/ Göttler, E., Phil.Mag. 28 (1973) 1057 .

Note 1. According to its meaning $\tau$ does not contain an athermal contribution $\tau_{i}$, of course. For a finite $\tau$ it is $\tau_{00}=\tau_{0}(0)-\tau_{i} / 27 /$. See note 2 .

Note 2. The parameters are deduced /27/ utilizing not $\tau(T)$ itself, but its derivative, $d \tau_{0} / d T$. In this way a value for a possibly existing athermal contribution $\tau_{i}$ to $\tau_{0}(T)$ has not to be arbitrarily assumed a priori. Rather, with the parameters determined, $\tau_{i}$ is calculated from $\tau_{0}(T)=\tau_{r}(T)+{ }^{i} \tau_{i}$.

In fact, it turns out as independent of temperature thus proving the procedure to be self-consistent. 